In: Found. Physics., Vol. 15, No. 1, (1983) 63-87.

\title{
Quantum Mechanics from Self-Interaction
}

\author{
David Hestenes
}

We explore the possibility that zitterbewegung is the key to a complete understanding of the Dirac theory of electrons. We note that a literal interpretation of the zitterbewegung implies that the electron is the seat of an oscillating bound electromagnetic field similar to de Broglie's pilot wave. This opens up new possibilities for explaining two major features of quantum mechanics as consequences of an underlying physical mechanism. On this basis, qualitative explanations are given for electron diffraction, the existence of quantized radiationless states, the Pauli principle, and other features of quantum mechanics.

\section{INTRODUCTION}

The theory of electrons is the workshop where quantum mechanics was designed, built, and tested. It culminates in the Dirac theory, which has survived every experimental test and produced many of the most precise and surprising predictions of quantum mechanics. Accordingly, any assessment of quantum mechanics, including its physical interpretation and any proposed extensions or modifications, should begin with an assessment of the Dirac theory. Why does the Dirac theory work so well? What are its limitations, and how might it be extended and improved?

For purposes of our discussion, the Dirac theory is to be understood as a theory of the electron with electromagnetic interactions. Mathematically, it consists of the Dirac wave function and its properties, including the Dirac equation and relations to physical variables such as energy-momentum, spin, and position probability current. Second quantization will not be regarded here as part of the Dirac theory, though this need not limit us to a one-particle interpretation of the theory.

As the basis for a satisfactory physical interpretation of quantum mechanics, we need a complete and coherent interpretation of the Dirac theory. An interpretation is complete if it provides a physical interpretation for every significant feature of the mathematical theory. It is coherent if the interpretation for each feature of the theory fits naturally into a unified interpretation of the whole. In spite of its indisputable mathematical successes, the Dirac theory is still without a completely satisfactory physical interpretation. In particular, the so-called zitterbewegung is a distinctive feature of the Dirac theory which continues to be the subject of conflicting interpretations in the literature.

This article aims to show that a complete and coherent interpretation of the Dirac theory can be achieved by interpreting the zitterbewegung literally as a point particle motion. The interpretation of the zitterbewegung suggested here is significantly different from Schroedinger's, and it has the advantage of providing a coherent interpretation for all the parameters in the Dirac theory. Moreover, it has implications that have not been previously discussed. In particular, it implies that the electron is the seat of a fluctuating electromagnetic field which oscillates with the de Broglie frequency of the electron. Thus, it tells us 
that a kind of electromagnetic wave-particle duality is implicit in the Dirac theory. Of course, this contradicts the conventional view that wave-particle duality is a property of matter which is completely independent of the nature of interactions. To show that the idea of electromagnetic wave-particle duality is not altogether unreasonable, we use it to provide qualitative explanations for the most distinctive features of quantum mechanics, from quantization and diffraction to spin and the Pauli principle.

Nothing is free. The price of a complete and coherent explanation of quantum mechanics based on the literal zitterbewegung idea is speculation about physical mechanisms that lie outside of established theory. We see at the beginning of our analysis that if the szitterbewegung is truly an objective phenomenon, then it almost certainly originates from electron self-interaction, since it persists in the absence of external fields. Consequently, a quantitative evaluation of the zitterbewegung idea cannot be achieved without solving the self-interaction problem.

Lacking that momentous theoretical breakthrough, we must be content with a qualitative account of the zitterbewegung which has a significant speculative component. This falls short of a theory which we can believe with much confidence. But it does outline a fairly definite research program for developing a complete theory, including specific hints about what is needed to solve the self-interaction problem. The reader will decide whether research in this direction is worthwhile.

\section{THE ZITTERBEWEGUNG}

The concept of zitterbewegung ( $z b w)$ was introduced by Schroedinger ${ }^{(1)}$ to interpret highfrequency oscillations in free-particle wave packets of the Dirac theory. These oscillations with angular frequency $2 m c^{2} / \hbar$ arise from interference between positive and negative energy components of a wave packet. Schroedinger interpreted the oscillations as fluctuations in positions of the electron about an average motion. Thus, his work raised the question: "What objective physical significance can be attributed to the zbw?" Three different answers can be found in the literature:

A. The $z b w$ is a mathematical artifact of the one-particle Dirac theory which does not appear in a correctly formulated quantum field theory.

B. The $z b w$ is an erratic motion of the electron due to random electron-positron pair creation and annihilation.

C. The $z b w$ is a localized helical motion of the electron with an orbital angular momentum which can be identified with the electron spin.

Of course, answer A amounts to a denial that the $z b w$ has any physical significance. It might be regarded as the majority view among the many practitioners of quantum field theory who do not mention the zbw. Although this answer is not without problems, it will not be of interest to discuss the matter here.

Answer B is sometimes offered as an explanation for the interference between positive and negative energy states. ${ }^{(2)}$ However, no attempt has been made to explain why random pair creation should produce regular oscillations. Nor does the idea play any role in actual 
applications of the theory.

Arguments in support of answer $\mathrm{C}$ have been developed by Huang ${ }^{(3)}$ and, more recently, by Barut and Bracken. ${ }^{(4)}$ Unfortunately, they do not explain why a helical motion or spin should depend on interference between positive and negative energy states or why the $z b w$ should depend on the way a wave packet is constructed. ${ }^{(5)}$ How can the $z b w$ be the origin of electron spin when it vanishes for plane wave states? For the plane wave functions certainly describe a particle with spin.

In spite of the inconclusive supporting arguments, answer $\mathrm{C}$ is worth serious consideration, because it proposes a physical explanation for electron spin. Surely, if answer C is correct, then the $z b w$ must be a fundamental property of electron motion. The inconclusive arguments show that a satisfactory characterization of the $z b w$ cannot be derived from the Dirac theory as it stands. So if we take the $z b w$ seriously, we must reverse the arguments. We must assume that the $z b w$ is an objective property of electron motion which underlies the Dirac theory. To do this we must go beyond the Dirac theory in assigning properties to characterize the $z b w$, but we must do it in a way which is consistent with the success of the Dirac theory.

If the $z b w$ is indeed a fundamental property of electron motion, then it should provide an interpretation for any electron wave function, plane waves as well as wave packets. We can arrange this by identifying the oscillatory motion of the $z b w$ with oscillations in the phase of the Dirac wave function. Although this differs somewhat from Schroedinger's original idea, it is not unrelated, for oscillations of the interference terms in a wave packet are produced by phase oscillations of the component plane waves. This idea will guide our characterization of the $z b w$ in the next section.

\section{PARTICLE PROPERTIES OF THE ELECTRON}

Our task now is to construct the simplest conceivable model of the $z b w$ consistent with the Dirac theory. We need a model which accounts for the electron's mass, spin, and periodicity. We begin with the idea that the electron spin is an orbital angular momentum in some instantaneous inertial system, call it the rest system of the electron. The simplest realization of this idea is a massless point particle executing circular motion in the rest system. The radius of the circular orbit will be called the $z b w$ amplitude, while the circular frequency of the motion is called the $z b w$ frequency. The center of orbit will be called the $z b w$ center, and the angular position of the electron on the circular orbit will be called the zbw phase angle.

Agreement with the physical parameters in the Dirac theory can be achieved if we assume that our model electron has the following properties:

(1) The electron is a massless point particle.

(2) The electron undergoes zbw with an intrinsic orbital angular momentum or spin of fixed magnitude $s=\hbar / 2$. 
(3) The $z b w$ frequency can be identified with the electron de Broglie frequency

$$
\omega_{0}=m c^{2} / \hbar=7.8 \times 10^{20} \mathrm{~s}^{-1}
$$

(4) The electron has an electric charge $e$.

(5) The total free electron self-energy is given by

$$
m c^{2}=E_{0}+U_{0}
$$

where $E_{0}$ is the $z b w$ kinetic energy and $U_{0}$ is the potential energy of self-interaction.

From these properties, a number of other properties can be derived. Since the electron is massless, it moves with the speed of light $c$, so property (3) implies that the zbw amplitude $r$ is equal to the electron Compton wavelength,

$$
r=c / \omega_{0}=\hbar / m c=3.8 \times 10^{-13} \mathrm{~m}
$$

Property (2) implies that $s=r\left(E_{0} / c\right)=\hbar / 2$; hence, the intrinsic zbw kinetic energy is

$$
E_{0}=s \omega_{0}=m c^{2} / 2
$$

Combined with property (4), this implies

$$
U_{0}=E_{0}=m c^{2} / 2
$$

Therefore, the total self-energy consists of equal parts of kinetic and potential energy.

It is reasonable to consider alterations of the model to see if the entire self-energy $m c^{2}$ can be identified with the $z b w$ kinetic energy, but this runs afoul of another property of the electron. Since the electron is charged, the $z b w$ motion generates a magnetic moment of magnitude

$$
\mu=(e c / 2 \pi r)\left(\pi r^{2} / c\right)=e r / 2
$$

This agrees with the result from the Dirac equation,

$$
\mu=\frac{e}{m c} s=\frac{e \hbar}{2 m c}
$$

only if $r=\hbar / m c$, as above. We cannot alter $E_{0}$ without altering this result. It should be noted, also, that the sign of the charge determines the relative directions of spin and magnetic moment, thus providing a physical distinction between the $z b w$ of electrons and positrons.

We have designed our electron model to give a simple, consistent, and coherent account of the physical parameters in the Dirac theory, namely, the mass, spin, magnetic moment, and de Broglie frequency of an electron. We shall employ the model to interpret the Dirac wave function and quantum mechanics more generally, but first we should consider its characteristics in more detail.

The model tells us that the world line of an electron in space-time is a helical curve winding around a timelike line traced out by the $z b w$ center. This $z b w$ centerline is a kind of 
average world line of the electron. The electron's instantaneous kinetic energy-momentum vector $P$ is a lightlike vector tangent to the electron world line. If the curvature of the centerline is not too great, an energy-momentum vector $p$ tangent to the $z b w$ centerline can be defined by

$$
p=2\langle P\rangle \text { ave }
$$

where the average is taken over a $z b w$ period. This vector defines an instantaneous rest frame for the electron. In accordance with our assumptions above, we identify $p$ as the total self-energy-momentum vector of the electron, and we have $p^{2}=m^{2} c^{2}$.

In the absence of external fields, $p$ is constant and the $z b w$ centerline is a straight timelike line. The $z b w$ persists, however; so if we wish to explain why the electron behaves so strangely, there appears to be no more likely option than to attribute the $z b w$ to electromagnetic self-interaction. Thus, we surmise that the electron mass and spin can be identified with the energy and angular momentum of electromagnetic self-interaction.

This puts the electron self-interaction problem in a new light. It tells us that the selfenergy problem cannot be solved without solving a related self-angular momentum problem. It suggests that the self-interaction is such that there exist certain stable, nonradiating but accelerated states of motion; in particular, for a free particle, motion in a circle with radius of a Compton wavelength - the zbw. The idea that the electron mass is due to electromagnetic self-interaction has been a favorite of theoreticians since the first theory of electrons by Lorentz. But it has usually been assumed that the self-energy is entirely potential energy. On the contrary, the $z b w$ implies that some of the mass, at least, is kinetic self-energy associated with a spin, and it reconciles the mass with a zero mass of the bare electron. Indeed, the fact that the electron and muon have the same spin but different masses suggests that spin rather than mass is the most characteristic feature of $z b w$ and self-interaction. If this view is on the right track, then the self-interaction problem cannot be solved with conventional quantum electrodynamics (QED). Rather, QED presupposes a solution of the self-interaction problem. The self-interaction problem must be solved to derive the Dirac theory on which QED is based.

The idea that the electron spin arises from a helical world line in spacetime has been considered before by many people (see Ref. 6 for an extensive account and further references). But it has not previously been related to the electron mass and phase to produce a complete interpretation of the Dirac theory. The idea of deriving electron spin from the radiative reaction (i.e., self-interaction) has been considered by Browne ${ }^{(7)}$ and Barut. ${ }^{(8)}$ Unfortunately, the classical arguments they employ cannot produce the properties of spin and $z b w$ needed to explain the Dirac theory without some profound modifications.

To solve the self-interaction problem and explain the $z b w$, it will presumably be necessary to begin with a suitable electron equation of motion coupled to electromagnetic field equations. No attempt to divine such equations will be made here. We only suggest that any formulation of the $z b w$ should be regarded as a clue to a deeper dynamical theory of electrons.

In the presence of an external field, the $z b w$ centerline will be curved rather than straight, but the electron spirals around it just the same. Of course, we would need an equation of motion to deduce the curvature of the centerline. But, for the purpose of interpreting the Dirac theory, it is sufficient to take the $z b w$ for granted, as we shall see in the next section. Then the Dirac equation can be used as the equation of motion. We are not yet in a position to derive the Dirac equation from a deeper dynamical theory. 


\section{INTERPRETATION OF THE DIRAC WAVE FUNCTION}

The reader is assumed to be familiar with the standard mathematical formulation of the Dirac theory. ${ }^{(9)}$ We aim to supply the theory with a physical interpretation by providing a complete and coherent interpretation of the Dirac wave function in terms of the $z b w$.

A physical interpretation of the electron wave function $\Psi$ in the Dirac theory is specified by the following three postulates:

I. The electron is a point particle undergoing zbw, and $\widetilde{\Psi} \gamma_{0} \Psi=\Psi^{\dagger} \Psi$ is the probability density for the position of the $z b w$ center in a given inertial system. Accordingly, the Dirac current

$$
J^{\mu}=\widetilde{\Psi} \gamma^{\mu} \Psi
$$

specifies the most probable direction of the $z b w$ centerline at each space-time point.

II. The spin density

$$
s^{\mu}=\frac{i \hbar}{2} \widetilde{\Psi} \gamma_{5} \gamma^{\mu} \Psi
$$

specifies the most probable direction of the $z b w$ spin at each space-time point.

III. The phase of $\Psi$ at each space-time point specifies the most probable $z b w$ phase of the electron moving with zbw center at that point.

Postulate I is a slight variant of the Born statistical interpretation of the wave function. The Dirac equation implies the conservation law $\partial_{\mu} J^{\mu}=0$, so $J^{\mu}$ determines a family of streamlines in space-time and a probability that the electron's $z b w$ centerline can be identified with any given streamline. Postulates II and III assign a spin direction and phase to the $z b w$ at each point on a given streamline, and the Dirac equation implies that spin and phase vary smoothly along the streamline. Therefore, we may imagine the electron's world line as a lightlike spiral of radius $\hbar / m c$ about a streamline determined by the Dirac equation.

The amplitude $\hbar / m c$ of $z b w$ fluctuations in position is not specified by the wave function, but it is determined by the Dirac equation in accordance with our interpretation. Later we ascribe uncertainty relations and the Lamb shift to these fluctuations.

As a simple example of great importance, consider the interpretation of a polarized plane wave

$$
\Psi=u e^{-i p \cdot x / \hbar}
$$

The Dirac equation implies that $p^{2}=m^{2} c^{2}$, and

$$
J^{\mu}=\tilde{u} \gamma^{\mu} u=p^{\mu} / m c
$$

is a constant vector. Therefore, $p$ can be identified with the electron self-energy-momentum vector introduced previously. The direction of $p$ is the direction of the $z b w$ centerline in space-time. The uniform magnitude of $\Psi$ and $J^{\mu}$ means that all timelike lines with the direction of $p$ are equally probable space-time trajectories for the $z b w$ center. The electron spin

$$
s^{\mu}=\frac{i \hbar}{2} \tilde{u} \gamma_{5} \gamma^{\mu} u
$$


is a constant vector with magnitude $\left|s^{\mu}\right|=\hbar / 2$ and direction orthogonal to $P$. Therefore, the electron has a fixed spin $s$, whatever its centerline trajectory. The phase of the wave function at a space-time point $x$ has the value

$$
\phi=\frac{p \cdot x}{\hbar}=\frac{m c^{2}}{\hbar} \tau=\omega_{0} \tau
$$

where $\tau$ is the proper time along a $z b w$ center line, or Dirac streamline if you will. This agrees exactly with the $z b w$ phase angle introduced earlier. Thus, the $z b w$ provides a complete interpretation for a polarized plane wave function.

For an arbitrary wave function, the physical interpretation specified by our three postulates is not quite complete. Eight real parameters are required to specify the wave function $\Psi$. Four of the parameters determine the Dirac current $J^{\mu}$, which, in turn, determines their interpretation. One of these four parameters determines the probability density, while three parameters determine the direction of the Dirac current. Since $s_{\mu} J^{\mu}=0$, only two more parameters are needed to determine the spin direction. Of course, the phase of the wave function is itself a single real parameter. Thus, the three postulates specify a physical interpretation for seven of the eight parameters in $\Psi$. For plane wave states, the remaining parameter distinguishes electron and positron states, but its physical significance in general is difficult to ascertain. This parameter is explicitly identified in the Appendix, where the wave function is represented in a form which clarifies its physical interpretation.

To complete the physical interpretation of the Dirac theory, we must specify how the energy-momentum of the electron is related to the wave function in general. We can do this by adopting the standard prescription identifying $i \hbar \partial_{\mu}$, as an energy-momentum operator. To see how this relates to the $z b w$, consider the equation

$$
i \hbar \partial_{t} \Psi=E \Psi
$$

which states that the electron has a definite total energy $E$ in a given inertial system. Note the operator extracts the energy. This tells us that the zbw has a frequency $\omega=E / \hbar$ in the given inertial system. It also tells us that the $z b w$ frequency is determined by the total energy and not the self-energy alone as in our initial description of the $z b w$. The self-interaction determines a minimum electron mass, but the mass is increased by external interactions. We have seen that the $z b w$ amplitude is inversely proportional to the mass, and the Dirac theory tells us that the magnitude of the spin is unaffected by external interactions. Therefore, external interactions will reduce the $z b w$ amplitude, or if you will, the "size" of the electron. The reduction is relatively small for typical atomic interactions, but it should contribute to the anomolous magnetic moment of the electron. All this suggests that the great importance of the operator $i \hbar \partial_{\mu}$ in quantum mechanics derives from the fact that it relates energy-momentum to the $z b w$ phase and amplitude. 


\section{WAVE PROPERTIES OF THE ELECTRON}

Our description of the electron has so far ignored the fact that the electron is the seat, or source if you will, of an electromagnetic field. The field of a point charge executing $z b w$ differs from the field usually attributed to an electron. The $z b w$ is a circular motion in the electron's rest system. This motion produces an oscillating electromagnetic field with the same frequency as the zbw, about $10^{21} \mathrm{~Hz}$. The field oscillates about a static average field obtained by averaging over a period of the $z b w$. Since the $z b w$ amplitude is so small, to a good approximation, the time average electric field of an electron is equal to the coulomb field of a point charge located at the $z b w$ center. We have already determined that the $z b w$ produces a magnetic moment in agreement with the Dirac theory. Therefore, we can conclude that the time average magnetic field of the electron is equal to the field of a magnetic dipole of strength $\mu=e \hbar / 2 m c$ located at the $z b w$ center.

The time average electric and magnetic fields produced by the $z b w$ are the fields usually attributed to an electron. But the $z b w$ implies in addition that the electron is the seat of a bound electromagnetic field oscillating with the de Broglie frequency and wavelength of the electron. This oscillating field is a kind of electromagnetic de Broglie wave, so let us adopt a term of de Broglie's and call it the pilot wave of the electron. We may think of the complete electron as a point particle with a pilot wave attached to it. Thus, the $z b w$ implies a kind of electromagnetic wave particle duality where the electron is both wave and particle.

Although the pilot wave does not appear in the conventional Dirac theory, we shall see that it is not only consistent with the Dirac theory but provides us with a physical mechanism for explaining some of the most mysterious features of quantum mechanics. The explanatory power of the pilot wave concept is largely due to the fact that the pilot wave and the Dirac wave function have a critical property in common. At every spacetime point the phase of an electron's pilot wave has the same value as the phase of the Dirac wave function for the electron, subject to suitable initial conditions. This supplies a physical basis for the smooth phase function that the wave function associates with an electron. It tells us that some wave properties of the physical pilot wave are represented by wave properties of the statistical wave function.

A stationary oscillating field like the pilot wave does not appear in classical electrodynamics, because the classical theory implies that an isolated accelerating charge must radiate. This is not a consequence of Maxwell's equations, however, but of the classical equations of motion and the coupling of source to field. These features of the classical theory cannot be completely correct, because they lead to unsatisfactory results in the self-interaction problem. ${ }^{(10)}$ Why is the kinetic energy and angular momentum in the $z b w$ not rapidly radiated away? That is part of the self-interaction problem. If the $z b w$ exists, then the pilot wave must exist, and the fact that it is a nonradiating oscillatory field bound to the electron should be regarded as a clue to the solution of the self-interaction problem. This suggests that the self-interaction is such that there exist certain stable, nonradiating but accelerated states of motion; in particular, for a free particle, motion in a circle with radius of a Compton wavelength - the $z b w$. We shall see that the $z b w$ provides a mechanism for producing other quantized nonradiating states of motion in external fields.

The foregoing suggests that the difference between classical and quantum electrodynamics lies not in the properties they attribute to the electromagnetic field but ultimately in the 
treatment of self-interaction. Accordingly, we suppose that the electromagnetic field in QED is completely described by Maxwell's equations, just as in the classical theory. We maintain that quantization of the electromagnetic field is a superfluous feature of QED. As justification for this position, we shall explain how physical effects commonly attributed to quantization of the electromagnetic field can be alternatively understood as consequences of the zbw.

\section{INTERPRETATION OF QUANTUM MECHANICS}

We have completed our formulation of the $z b w$ and the interpretation of the Dirac theory. Now we use these ideas to provide qualitative explanations for the most distinctive features of quantum mechanics. These features are largely mathematical consequences of the Dirac theory, but explanations based on the $z b w$ call for a dynamical theory operating at a deeper level.

\subsection{Spin-Uncertainty Relations}

If we wish to localize a free electron, the $z b w$ implies that the best we can do is confine it to a circular orbit of radius $r=\hbar / m c$ with a fixed center. Therefore, the $x$-coordinate of the electron in the orbital plane will fluctuate with a range $\Delta x=\hbar / m c$. At the same time, since the electron travels at the same time, and since the electron travels at the speed of light with a zeropoint kinetic energy $m c^{2} / 2$, the $x$-component of its momentum fluctuates with a range $\Delta p_{x}=m c / 2$. Thus, we obtain the minimum uncertainty relation

$$
\Delta x \Delta p_{x}=\hbar / 2
$$

We now see the uncertainty relations as consequences of a zero-point motion with a fixed zero-point angular momentum, the spin of the electron. This explains why the limiting constant $\hbar / 2$ in the uncertainty relations is exactly equal to the magnitude of the electron spin.

\subsection{Diffraction}

Any coherent interpretation of quantum theory must give a satisfactory account of electron diffraction. Two major alternative have been proposed in the past: the conventional dualistic interpretation and the particle interpretation espoused by the late Alfred Landé. ${ }^{(11)}$

The conventional account is based on the premise that diffraction can only be understood as the result of wave interference. Accordingly, it is supposed that the electron must be regarded as a wave when undergoing diffraction. On the other hand, electrons are detected only as particles in a diffraction pattern. It is claimed that this forces one into the dualistic view that the electron is neither wave not particle, but manifests itself as one or the other depending on circumstances. As de Broglie ${ }^{(12)}$ has emphasized, a serious difficulty with the dualistic account of diffraction is that it uses the wave concept in two different senses. 
The wave undergoing diffraction belongs to a single electron, but the wave manifested in the observed diffraction pattern is clearly a property of the ensemble of electrons in the pattern.

Landé contends that difraction can be explained as quantized momentum exchange without regarding the electron as a wave. His view is consistent with the fact that, in modern many-body theory, diffraction patterns can be calculated from symmetry properties of a crystal without using the idea of interference. The discrete spectrum of vibrational states in a crystal is determined by symmetry properties of the crystal wave function, and the Bragg diffraction formula is determined by the allowed transitions between those states. Thus, the crystal acting as a unit can exchange only quantized amounts of momentum with an electron or any other particle or wave it might scatter. A difficulty with Landé's account is that it does not explain how a localized electron can induce an extended crystal to act as a unit.

The $z b w$ provides us with a new way to explain electron diffraction which accounts for both wave and particle aspects of the phenomena. An electron incident on a crystal is surrounded by its pilot wave which begins to drive charges in the crystal even before the electron arrives. The driven charges in the crystal act back on the electron, and momentum transfer will be greatest at resonances corresponding to transitions to allowed excitations of the crystal. Thus, the pilot wave provides the mechanism for momentum transfer by a particle which is missing from Landé's explanation. To establish this explanation as completely viable, it will be necessary to derive the Bragg laws and diffraction intensity patterns by direct application of the pilot wave mechanism rather than indirectly from the wave equation.

It is of interest to note that de Broglie has argued at length that a satisfactory interpretation of quantum theory cannot be achieved without postulating the existence of physical "pilot waves" which are distinct from quantum mechanical wave functions. ${ }^{(12)}$ Our pilot wave differs from his in being specifically electromagnetic and related to the electron spin. Also de Broglie explained electron diffraction as due to interference of the pilot wave which, in turn, "guides" the electron to the diffraction pattern. In our explanation the electron plays a more active role, and it might be better to say that, instead of being guided, the electron carries the pilot wave with it, although the pilot wave arrives first to announce the coming electron.

\subsection{Universality of Wave-Particle Duality}

Diffraction of material particles is a universal phenomenon. The conventional explanation for this fact is that each material particle possesses a wave property which is completely independent of its interactions. In contrast, our pilot wave explanation for electron diffraction is manifestly electromagnetic. A similar explanation for the diffraction of neutrons, atoms, and molecules obtains only if every material system is the seat of an electromagnetic pilot wave with de Broglie frequency and wavelength determined by its mass. We shall see that this is to be expected for atomic systems composed of electrons. We take it as a clue to the structure of elementary particles like the neutron to be discussed later. 


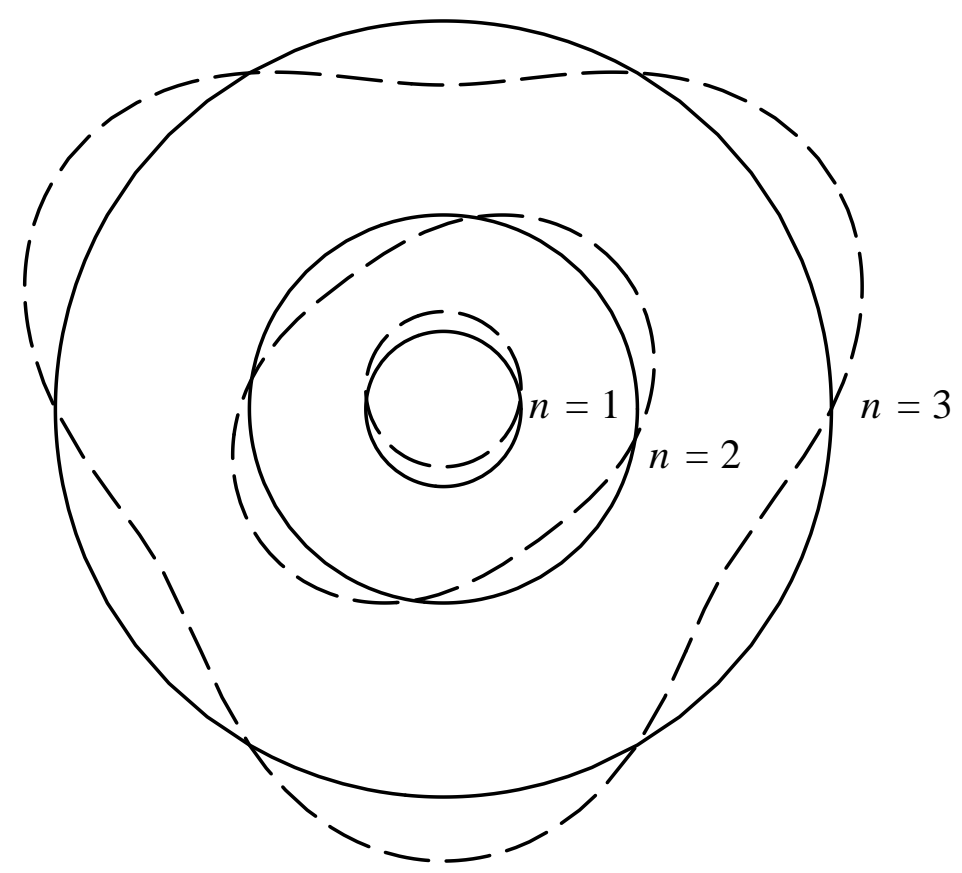

Fig. 1. Zbw oscillations of a bound electron.

\subsection{Stationary Quantized States}

The conventional interpretation of the quantum theory does not explain the existence of stationary quantized atomic states beyond the fact that they correspond to stationary solutions of the Dirac equation. The $z b w$ interpretation enables us to do better, as it should if it is correct. It will suffice for us to discuss stationary states of the hydrogen atom with the nucleus regarded as a point charge.

The stationary states of the hydrogen atom correspond to eigenstates of the energy operator. Hence, the $z b w$ of a bound electron has a definite frequence $\omega=E / \hbar$ : the stationary wave functions are single-valued in space, so the phase of the wave function is single-valued as well. Since the phase of the wave function corresponds to the phase of the electron $z b w$, it follows that in a stationary atomic state the orbital period is an integral multiple of the electron $z b w$ period, that is, there is a coherence between the $z b w$ and the orbital motion. This is represented schematically in Fig. 1, showing $z b w$ oscillations of the "true" electron orbits (dotted lines) about circular orbits of the $z b w$ center for the first three allowed states. The figure looks just like a representation of de Broglie standing waves in Bohr orbits for the good reason that it conforms to de Broglie's original idea that the stationary orbits are determined by an intrinsic periodicity in the electron motion. Of course, the representation of the periodicity is different here. The waves in Fig. 1 are to be interpreted literally as orbits, and the $z b w$ amplitude of oscillation has a definite value on the order of an electron Compton wavelength.

The association of the single-valuedness of stationary state wave functions with the coherence of orbital and $z b w$ motions allows us to explain the former in terms of the latter. At the same time we wish to explain why stationary atomic states do not radiate. The 
conventional quantum mechanical treatment of the hydrogen atom arbitrarily takes the electron as a test charge, ignoring its field altogether, so it cannot radiate. This was always regarded by Schroedinger as a major failing of quantum mechanics. ${ }^{(13)}$ It is true that QED includes the electron field in perturbative corrections, but that does not account for the stability of the unperturbed state.

From the beginning of his research on quantum wave equations, Schroedinger sought to explain the existence of quantized radiationless states as the result of some kind of resonance. The $z b w$ gives us a mechanism for doing that. The electromagnetic field of an electron orbiting a nucleus can be separated into two periodic components, the pilot wave due to its $z b w$, and the average field due to its orbital motion. We can guess that the resultant field will be radiationless when these fields are resonant, and we know that this corresponds to the condition for stationary states, but a proof that such a field will be radiationless cannot be supplied until the self-interaction problem has been solved. At least we have a qualitative explanation for the existence of radiationless states. This explanation also implies that the oscillation of a bound electron produces an oscillating electromagnetic field which surrounds the atom and has a frequency determined by the energy of the bound state. This field is the pilot wave of the atom. The long-range tail of this fluctuating field has been identified with the Van der Waals force. ${ }^{(14)}$

\subsection{The Pauli Principle}

In calculating the energy of a many-electron atom the electrostatic and magnetic interactions between electrons are taken into account. But according to our theory these are not the only interactions, for the $z b w$ of each electron produces an oscillating field as well. The effect of such fields would certainly be to introduce correlations in the positions and spins of the electrons, and the allowed combinations of electron orbits would be restricted to those which produce stable radiationless states. This, in turn, would be reflected in symmetry properties of the many-electron wave function such as those required by the Pauli principle. Thus, we have a physical mechanism for explaining the Pauli principle: The Pauli principle describes symmetry properties of a many-electron configuration which result from stable resonances of their pilot waves. The symmetries are spin dependent, because the pilot waves are spin dependent. The Pauli principle works only for like particles, because only like particles have the identical frequencies needed to produce a resonance. The so-called exchange force that results from the Pauli principle can thus be understood as physically real force due to oscillations of the pilot waves. Its origin is therefore basically the same as that of the Van der Waals force.

\subsection{The Lamb Shift and Spontaneous Emission}

Many physicists follow Welton ${ }^{(15)}$ in attributing the Lamb shift to fluctuations of the electromagnetic vacuum field. Welton argues that the effect of the fluctuations is to smear out the point electron over a region about the size of a Compton wavelength. This produces a shift in potential energy depending on the average of the potential over the region.

The zbw gives us a different interpretation of the Lamb shift. The potential energy 
assigned to the electron by the Dirac theory is the value at the zbw center. This differs from the correct potential at the position of the electron by an amount which oscillates over a Compton wavelength, as indicated in Fig. 1. Thus we attribute the "smearing out" of the electron over a Compton wavelength to the systematic zbw oscillations rather than random fluctuations of the vacuum field. Of course, we are speaking here of the main contribution to the Lamb shift. In addition, a small contribution should come from a shift in the $z b w$ amplitude, which we have already mentioned as a source of the anomalous magnetic moment. Of course, we may also expect contributions from many particle effects such as vacuum polarization.

A number of physicists (in particular, Jaynes, ${ }^{(16)}$ and Barut and $\mathrm{Kraus}^{(17)}$ ) have argued that the Lamb shift is due to radiative reaction. That viewpoint is closely related to ours, since the radiative reaction is a consequence of electromagnetic self-interaction, and we have suggested that self-interaction is the origin of the zbw. This brings us back again to the problem of determining a form of the self-interaction consistent with quantum theory.

Besides the Lamb shift, spontaneous emission is commonly attributed to the electromagnetic vacuum field. The idea is that the fluctuating vacuum field stimulated emission from excited atomic states. On the other hand, in classical theory the damping of an oscillating dipole is due to radiative reaction, and the same mechanism can be used to account

for spontaneous emission in quantum theory. ${ }^{(16)}$ Milonni and Smith ${ }^{(18)}$ have shown that in quantum field theory spontaneous emission can be attributed either to radiative reaction or to vacuum fluctuations, depending on the ordering of field operators. Barut ${ }^{(19)}$ attributes spontaneous emission to the $z b w$.

All this suggests that the electromagnetic vacuum field is an artifice of quantum field theory which simulates certain effects whose real physical origin is quite different, namely, the $z b w$, or the radiative reaction, if you will. This brings the quantization of the electromagnetic field into question, since that is what introduces the vacuum field into QED, and the vacuum field is chiefly responsible for the physical consequences of quantization. The position taken here is that quantization is something to be explained rather than assumed. We have seen how pilot waves regarded as classical fields might provide a plausible explanation for quantized atomic states. The existence of quantized states implies quantized emission and absorption of electromagnetic energy, so no additional quantization of fields seems necessary. This quantization issue is discussed at greater length in Ref. 14 .

\section{THE STRUCTURE OF ELEMENTARY PARTICLES}

If the $z b w$ is the key to the Dirac theory, then surely it is a key to the structure of elementary particles. So let us consider, briefly, some of the possibilities opened up by the $z b w$ concept, though this may look like wild speculation from a conventional perspective.

Let us begin by noting that Asim Barut has mustered a collection of ingenious arguments contending that weak and strong interactions are actually short-range magnetic interactions. ${ }^{(20)}$ Since electrodynamics also plays a preferred role in our version of the $z b w$, it is interesting to speculate on possible connections with Barut's theory.

We explain the properties of the Dirac electron as consequences of the $z b w$, which is presumably due to electromagnetic self-interaction. To go beyond electron theory we must ask how the same $z b w$ mechanism might explain the existence of other elementary particles. 
There are two natural possibilities.

Considering only geometrical features of the $z b w$, we note that the world line of the $z b w$ center is timelike for an electron, and there is one other possibility where the world line of the $z b w$ center is lightlike. This alternative could only be identified with the neutrino. Accordingly, we suppose that the world line of a free neutrino is a lightlike helical curve winding around a lightlike straight line. The wave function for a neutrino can be constructed in the same way as the wave function for an electron. Thus, the geometry of the $z b w$ yields exactly two kinds of elementary particles, the electron and the neutrino, and their antiparticles, which differ only in the "helicity" or "sense" of their helical world lines.

To have a coherent theory, we should certainly require that the neutrino's $z b w$ arise from electromagnetic self-interaction just like the electron's. In fact, the simplest theory would take the electromagnetic interactions of the neutrino and the electron to be identical, the two particles differing only by being in different self-interaction states. This would not necessarily conflict with the fact that the neutrino is a neutral particle. For we have seen that the Coulomb field of the electron is an average field in the rest frame of the zbw. But there is no such rest frame for the neutrino. The neutrino does not "stay put" long enough to produce an average electric field. Perhaps this implies that the neutrino will not "appear" to be charged. We do not have enough experience with "lightlike charged particles" to say for sure. At any rate it is clear that the neutrino will have a pilot wave and an average field something like that of a magnetic moment. This may be what is needed for Barut's theory. For Barut explains the weak interactions of the neutrino electromagnetically, by assuming that the neutrino has a small magnetic moment.

There is another way the $z b w$ might be related to different elementary particle states. The expression for the $z b w$ radius $r=\hbar / m c$ is consistent with the possibility that the electromagnd proton. He argues that the binding is due to short-range magnetic interactions. Our $z b w$ model brings the possibility of a new kind of bound state where the helical world line of one lepton is intertwined with the helical world line of another. For example, if we follow Barut and consider the $\pi^{-}$as a $\left(e^{-} \bar{\nu}\right)$ pair bound in this way, then we picture the $\bar{\nu}$ helix as winding around the $e^{-}$helix. In other words, the zbw center of the $\bar{\nu}$ follows the helical $e^{-}$world line. This kind of bound state must be qualitatively different from atomic bound states where the $z b w$ orbit of an electron is not penetrated by another particle. Perhaps this is a key to the qualitative difference between observed strong and electromagnetic interaction.

The $z b w$ provides a rationale for the electron and neutrino as elementary particles but not for the proton. On the other hand, there is abundant evidence that the proton is a composite particle, even though it is stable. With the lepton building blocks we have available, the combination most likely to produce a proton consists of two positrons and an electron. At least this combination is consistent with the data from deep inelastic electron-nucleon scattering experiments as reported by Barut. ${ }^{(20)}$ But why doesn't the electron annihilate one of the positrons? Perhaps because the three leptons are bound by an intertwined linking of their zbw orbits, and this binding is such as to prevent annihilation except under extreme conditions. This seems no more farfetched that explanations for quark confinement. And it has the very great advantage of explaining the apparent imbalance between matter and antimatter in the universe. For it tells us that there is an extra positron buried in every proton. Therefore, if the Universe is electrically neutral as it appears to be, then it contains exactly as many positrons as electrons. 


\section{CONCLUSIONS}

The aim of this article has not been to modify the mathematical structure of the Dirac theory, but to supply it with a complete and coherent physical interpretation based on the $z b w$ concept. That aim has been met with reasonable success. But the question remains whether the $z b w$ is an objectively real phenomenon or just a picturesque metaphor. We have seen that the $z b w$ is a mechanism with the potential for explaining all the distinctive characteristics of quantum mechanics. But the qualitative explanations adduced here must be supported by quantitative calculations before one can be confident of their validity.

Quantitative explorations of the $z b w$ can be carried out at two levels. At the most superficial level, the $z b w$ and pilot wave concepts would be taken for granted and used directly in calculations. The most interesting results that might be achieved at this level would be calculations of diffraction patterns and exchange forces using pilot waves. And if the $z b w$ idea is correct, one should be able to derive the Dirac equation by supplementing it with some statistical and dynamical assumptions.

At a deeper level one would seek to formulate a dynamical theory with self-interactions which produce the $z b w$. One can hope to achieve this by a suitable generalization of classical electrodynamics. Our qualitative discussion of pilot waves suggests that quantum theory differs from classical theory in the kinds of stable electromagnetic waves they admit. The classical theory admits only free electromagnetic waves representing electromagnetic radiation, whereas the quantum theory admits also bound (or standing) electromagnetic waves, the pilot waves attached to every particle. To realize this idea in a viable theory, it will be necessary to adduce an exact equation of motion for a single electron, including selfinteraction. This will require a solution of the self-interaction problem, which will probably not be convincing unless it produces a calculation of some fundamental constant like the fine structure constant or electron mass.

\section{APPENDIX: THE REAL ELECTRON WAVE FUNCTION}

In this appendix we discuss a representation of the Dirac wave function which is easier to interpret physically than the conventional matrix representation. We call it the real representation to emphasize the fact that it avoids the imaginary numbers employed in the matrix representation. The matrix formulation was used in our initial discussion of the interpretation of the wave function, because readers are most likely to be familiar with it. But the real formulation provides new insights into the structure of the wave function. Indeed, those insights provided the principal motivation for the developments in this article. Chief among these insights is the fact that the Dirac theory implicitly relates the unit imaginary number to the electron spin and so assigns it a physical interpretation. The real formulation given below makes this fact explicit. In view of the crucial role that complex numbers play in quantum mechanics, one might expect this fact to have some profound physical import. The $z b w$ interpretation in this article was developed in an attempt to divine that import. But the fact remains, and the real representation is important even if the interpretation is totally wrong.

The real representation of the Dirac wave function was discovered in Ref. 21, and its 
relation to conventional theory has been established in various ways in Refs. 22-24. The rationale for the representation will only be sketched here. The reader is referred to the above articles for full mathematical details.

Instead of the conventional Dirac algebra, we employ a real Clifford algebra called spacetime algebra (STA), because it can be interpreted as a mathematical grammar for describing space-time geometry. ${ }^{(25)}$ A Clifford algebra is simply an associative algebra generated by the elements of a vector space subject to the rule that the product of any vector with itself is scalar-valued. STA is the Clifford algebra generated by the vectors of four-dimensional Minkowski space-time.

With STA, an orthonormal frame of vectors $\gamma_{\mu}$ in space-time can be defined by the equations

$$
\gamma_{0}^{2}=-\gamma_{1}^{2}=-\gamma_{2}^{2}=-\gamma_{3}^{2}=1
$$

and

$$
\gamma_{\mu} \gamma_{\nu}=-\gamma_{\nu} \gamma_{\mu}
$$

for $\mu \neq \nu$ and $\mu, \nu=0,1,2,3$. These equations are similar to the well-known equations defining the Dirac matrices, except that the unit scalar 1 on the right side of (1) must be replaced by the identity matrix in the Dirac algebra. It follows that STA is isomorphic to the algebra generated by the Dirac matrices over the real numbers. The reason for replacing the Dirac algebra by STA in quantum theory is that the matrix representations and the imaginary scalars in the Dirac theory are totally irrelevant to space-time geometry as well as to the physical interpretation of electron theory. This is the crucial fact leading to a graphic interpretation of the electron wave function.

To explain how an electron wave function can be represented with STA, we need a little nomenclature and notation. A generic element of STA is called a multivector. Since STA is generated by vectors, every multivector can be expressed as a polynomial in the $\gamma_{\mu}$ with real coefficients. A multivector is said to be even if each nonvanishing term in the polynomial expansion is a product of an even number of vectors. From a multivector $M$ a "conjugate" multivector $\widetilde{M}$ is obtained by reversing the order of vectors in its polynomial expansion.

If $\psi$ is an even multivector, then we can always write

$$
\psi \widetilde{\psi}=\rho e^{\gamma_{5} \beta}
$$

where $\gamma_{5}=\gamma_{0} \gamma_{1} \gamma_{2} \gamma_{3}$ and $\rho$ and $\beta$ are scalars. If $\rho \neq 0$, then we can write $\psi$ in the canonical form

$$
\psi=\left(\rho e^{\gamma_{5} \beta}\right)^{\frac{1}{2}} R
$$

where $R$ is a unimodular even multivector in the sense that

$$
R \widetilde{R}=1
$$

We call $R$ a unimodular spinor if it is associated with a Lorentz transformation by the equations

$$
e_{\mu}=R \gamma_{\mu} \widetilde{R}
$$

These equations describe a Lorentz transformation of the orthonormal vectors $\gamma_{\mu}$ into an orthonormal frame of vectors $e_{\mu}$. In fact, every (proper) Lorentz transformation can be described in this way. ${ }^{(25)}$ From (4) and (6) it follows that

$$
\psi \gamma_{\mu} \widetilde{\psi}=\rho e_{\mu}
$$


Any even multivector $\psi$ determining a frame of vectors $\rho e_{\mu}$ in this way may be called a spinor. This definition is completely equivalent to the usual matrix definition of a Dirac spinor (except for a proviso when $\mid$ width0pt $\psi \widetilde{\psi}=0$ which need not concern us here). As in the matrix representation, the value of $\psi$ is determined by eight real parameters, but the canonical form (4) specifies a parametrization with geometrical meaning. Two of the parameters are exhibited explicitly as $\rho$ and $\beta$. The remaining six parameters determine $R$, or equivalently, the Lorentz transformation (6).

The electron wave function is a spinor-valued function $\psi=\psi(x)$ defined at each spacetime point $x$. The values of the wave function are determined by the Dirac equation, but we will be concerned here only with the physical interpretation assigned by the Dirac theory. The vector field

$$
J=\psi \gamma_{0} \widetilde{\psi}=\rho e_{0}
$$

can be identified with the Dirac current. The Dirac current is interpreted as a probability current. Hence the scalar-valued function $\rho=\rho(x)$ is an invariant probability density, and the vector field $e_{0}=e_{0}(x)$ describes the direction of probability flow in space-time. The vector field

$$
s=\frac{1}{2} \hbar \psi \gamma_{3} \widetilde{\psi}=\frac{1}{2} \hbar \rho e_{3}
$$

where $\hbar$ is Planck's constant, can be interpreted as a spin density, so $e_{3}$ specifies the electron spin axis. It should be understood that in this context $\left\{\gamma_{\mu}\right\}$ is an arbitrarily chosen fixed frame which need not be associated with an inertial coordinate system. So given $\left\{\gamma_{\mu}\right\}$, the spinor $\psi$ determines four orthogonal vector fields by (7). Only two of these vector fields, specified by (8) and (9), are identified and given a physical interpretation in the conventional matrix formulation of the theory.

Equations (8) and (9) for the Dirac current and spin density in terms of the real wave function $\psi$ are exactly equivalent to the conventional expressions in terms of the matrix wave function $\Psi$. The equivalence is expressed explicitly by

$$
J_{\mu}=\gamma_{\mu} \cdot J=\left(\gamma_{0} \widetilde{\psi} \gamma_{\mu} \psi\right)_{S}=\widetilde{\Psi} \gamma_{\mu} \Psi
$$

and

$$
s_{\mu}=\frac{\hbar}{2}\left(\gamma_{3} \widetilde{\psi} \gamma_{\mu} \psi\right)_{S}=\frac{i \hbar}{2} \widetilde{\Psi} \gamma_{5} \gamma_{\mu} \Psi
$$

where the subscript $S$ means scalar part, and the $\gamma_{\mu}$ in expressions on the right are understood to be matrix representations of the vectors $\gamma_{\mu}$.

Note that the parameter $\beta$ does not contribute to these physical quantities. Difficulties in assigning a physical interpretation to $\beta$ are discussed in Refs. 22 and 23 . Reference 23 suggests that $\beta$ might be explained as the result of constructing a wave function for a statistical ensemble of electron motions.

It will be noted how perfectly the physical interpretation of the wave function conforms to the geometrical interpretation. Five of the six parameters determining the Lorentz transformation (6) are required to determine the velocity and spin vectors $e_{0}$ and $e_{3}$. The remaining parameter is the phase of the wave function. Here we have a new insight, for the matrix formulation gives us no hint that the phase of the electron wave function is related to a Lorentz transformation. Moreover, this has bearing on the physical interpretation of electromagnetic gauge transformations. 
An electromagnetic gauge transformation induces a change in the wave function (4) described by

$$
\psi \rightarrow \psi^{\prime}=\psi e^{\gamma_{2} \gamma_{1} \phi}=e^{e_{2} e_{1} \phi} \psi
$$

where $\phi=\phi(x)$ is a scalar-valued "gauge function." The gauge transformation leaves the velocity and spin vectors $e_{0}$ and $e_{3}$ invariant, but it rotates $e_{1}$ and $e_{2}$ through an angle "about" the spin axis. Thus, a gauge transformation can be interpreted as a rotation in a spacelike plane at each point. According to (10), either $\gamma_{2} \gamma_{1}$ or $e_{2} e_{1}$ can be regarded as the generator of gauge transformations. They share the property of an imaginary unit: $\left(\gamma_{2} \gamma_{1}\right)^{2}=\left(e_{1} e_{2}\right)^{2}=-1$. Indeed, $\gamma_{1} \gamma_{2}$ takes over the role played by the scalar imaginary unit in the matrix formulation of the Dirac theory. Thus we see that imaginary numbers in the Dirac theory have a hidden geometrical significance.

Identification of the generator of electromagnetic gauge transformation with the generator of rotations in a spacelike plane has implications for the Weinberg-Salam model of electroweak interactions which have been discussed elsewhere. ${ }^{(27)}$ Here we consider a different possibility.

Since the Dirac current is a conserved vector field, it determines a family of timelike streamlines to which it is everywhere tangent. Following the evolution of the frame $e_{\mu}=$ $R \gamma_{\mu} \widetilde{R}$ along a streamline, we see that $e_{1}$ and $e_{2}$ rotate about the spin axis like the axes of a rigid body. This perfect analogy with relativistic rigid body motion is developed fully in Ref. 24. It applies to any solution of the Dirac equation. But it is easy to describe explicitly only for plane-wave solutions.

The Dirac equation yields exactly two distinct positive energy plane wave solutions for a particle with a definite proper momentum $p$ and spin polarization $e_{3}$. They are the electron plane wave $(\beta=0)$

$$
\psi_{-}=\rho^{\frac{1}{2}} R_{0} e^{-\gamma_{2} \gamma_{1} p \cdot x / \hbar}
$$

and the positron plane wave $(\beta=\pi)$

$$
\psi_{+}=\rho^{\frac{1}{2}} i R_{0} e^{+\gamma_{2} \gamma_{1} p \cdot x / \hbar}
$$

For both these solutions $\rho$ and $R_{0}$ are constant, $p^{2}=m^{2} c^{2}$, and

$$
\begin{aligned}
& \psi_{ \pm} \gamma_{0} \psi_{ \pm}=\rho R_{0} \gamma_{0} \widetilde{R}_{0}=\rho P / m c \\
& \psi_{ \pm} \gamma_{3} \psi_{ \pm}=\rho R_{0} \gamma_{3} \widetilde{R}_{0}=\rho e_{3}
\end{aligned}
$$

However,

$$
\psi_{ \pm} \widetilde{\psi}_{ \pm}= \pm \rho
$$

and, for $k=1,2$,

$$
\psi_{ \pm} \gamma_{k} \widetilde{\psi}_{ \pm}=\rho e_{k}=\rho e_{k}(0) e^{ \pm e_{2} e_{1} 2 p \cdot x / \hbar}
$$

where $e_{k}(0)=R_{0} \gamma_{k} \widetilde{R}_{0}$

Equation (13) tells us that the streamlines are straight lines tangent to $p$. If $\tau$ is the proper time along a streamline, with a suitable choice of origin we can write $p \cdot x=m \tau$ where

$$
\omega_{0}=m c^{2} / \hbar=7.8 \times 10^{20} \mathrm{~s}^{-1}
$$


is the de Broglie frequency along a streamline. Therefore,

$$
d \psi_{ \pm} / d \tau= \pm \frac{1}{2} \Omega \psi_{ \pm}
$$

and

$$
d e_{\mu} / d \tau= \pm \Omega \cdot e_{\mu}
$$

where

$$
\Omega=2 \omega_{0} e_{2} e_{1}=2 \omega_{0} R_{0} \gamma_{2} \gamma_{1} \widetilde{R}_{0}
$$

Equation (19) can be interpreted as the equation of motion for a frame $e_{\mu}$ moving along a streamline and rotating with an angular velocity $\pm \Omega$. The rotation is in a fixed plane characterized by the generator $\hat{\Omega}=e_{2} e_{1}$, with opposite senses for the electron and positron cases. The angular frequency of the rotation $|\Omega|=2 \omega_{0}$ is twice that of the wave function itself.

The notion that there is a periodicity of frequency $\omega_{0}=m c^{2} / \hbar$ associated with the motion of an electron was, of course, one of the original ideas of de Broglie ${ }^{(27)}$ leading to quantum mechanics. The Dirac equation incorporates this periodicity into the phase of the wave function. Now, by associating the frequency with a rotation about the spin axis, the real spinor representation of the wave function reveals a connection between the angular frequency (or mass) and the spin angular momentum. This fact provided the impetus for the interpretation of the Dirac theory developed in this article.

\section{REFERENCES}

1. E. Schroedinger, Sitzungb. Preuss. Akad. Wiss. Phys.-Math Kl. 24, 418 (1930).

2. H. Thirring, Principles of Quantum Electrodynamics (Academic Press, New York, 1958).

3. K. Huang, "On the Zitterbewegung of the Electron," Am. J. Phys. 20, 479 (1952).

4. A. O. Barut and A. J. Bracken, Phys. Rev. D 23, 2454 (1981).

5. J. A. Lock, "The Zitterbewegung of the Free Localized Dirac Particles," Am. J. Phys. 47, 797 (1979).

6. H. C. Corben, Classical and Quantum Theories of Spinning Particles (Holden-Day, San Francisco, 1968).

7. P. J. Browne, Ann. Phys. (N.Y.) 59, 254 (1970).

8. A. O. Barut, "What is an Electron?" in Quantum Electrodynamics in Strong Fields, W. Greiner, ed. (Plenum New York 1983).

9. J. D. Bjorken and S. D. Drell, Relativistic Quantum Mechanics (McGraw-Hill, New York, 1964).

10. F. Rohrlich, Classical Charged Particles (Addison-Wesley, Reading, Massachusetts, 1965).

11. A. Landé, Am. J. Phys. 33, 123 (1965); 43, 701 (1975); New Foundations of Quantum Mechanics (Cambridge University Press, Cambridge, 1965). 
12. L. de Broglie, The Current Interpretation of Wave Mechanics: A Critical Study (Elsevier, Amsterdam, 1964); Nonlinear Wave Mechanics: A Causal Interpretation (Elsevier, Amsterdam, 1960).

13. L. Halpern, personal communication.

14. D. Hestenes, "Spin and Uncertainty in the Interpretation of Quantum Mechanics," Am. J. Phys. 47, 399 (1979).

15. T. A. Welton, Phys. Rev. 74, 1157 (1948); J. D. Bjorken and S. D. Drell, Relativistic Quantum Mechanics (McGraw-Hill, New York, 1964), p. 58.

16. E. T. Jaynes, Coherence and Quantum Optics, L. Mandel and E. Wolf, eds. (Plenum, New York, 1978), pp. 495-509.

17. A. O. Barut and J. Kraus, "Nonperturbative Quantum Electrodynamics: The Lamb Shift," Found. Phys. 13, 189 (1983).

18. P. W. Milonni and W. A. Smith, Phys. Rev. A 11, 814 (1975).

19. A. O. Barut, "The Zitterbewegung and the Einstein A Coefficient of Spontaneous Emission," in Old and New Questions in Physics, Cosmology, Philosophy, and Theoretical Biology, A. van der Merwe, ed. (Plenum, New York, 1983).

20. A. O. Barut, "Stable Particles as Building Blocks of Matter," in Surv. High Energy Phys. 1(2), 113 (1980).

21. D. Hestenes, "Real Spinor Fields," J. Math. Phys. 8, 798 (1967).

22. D. Hestenes, "Local Observables in the Dirac Theory," J. Math. Phys. 14, 893 (1973).

23. D. Hestenes, "Observables, Operators, and Complex Numbers in the Dirac Theory," J. Math. Phys. 16, 556 (1975).

24. D. Hestenes, "Geometry of the Dirac Theory," in Mathematics of Physical SpaceTime, J. Keller, ed. (Facultad de Quimica, Universidad Nacional Autonoma de Mexico, Mexico City, 1981).

25. D. Hestenes, Space-Time Algebra (Gordon and Breach, New York, 1966).

26. D. Hestenes, "Space-time Structure of Weak and Electromagnetic Interactions," Found. Phys. 12, 153 (1982).

27. L. de Broglie, "Ondes et Quanta," Compt. Rend. 177, 507 (1923). 